\title{
Involucrando a profesores de primaria en el diseño de un juego serio mediante la metodología investigación-acción y co-creación
}

\author{
Ruth S. Contreras-Espinosa ${ }^{1}$, Jose Luis Eguia-Gómez ${ }^{2}$, Lluis Solano Albajes ${ }^{1}$ \\ ruth.contreras@uvic.cat, eguia@ege.upc.edu, solano@lsi.upc.edu \\ ${ }^{1}$ Universitat de Vic-Universitat Central de Catalunya-Facultad de empresa y comunicación, Sagrada Familia \\ 7, 08500, Vic \\ ${ }^{2}$ Universitat Politècnica de Catalunya- Departamento de expresión gráfica a la ingeniería, Av. Diagonal 647, \\ 08028, Barcelona \\ ${ }^{3}$ Universitat Politècnica de Catalunya- Departamento de lenguajes y sistemas informáticos, Av. Diagonal \\ 647, 08028, Barcelona
}

DOI: 10.17013/risti.20.115-130

\begin{abstract}
Resumen: Los juegos serios son herramientas que pueden ayudar al aprendizaje en escuelas. Este artículo describe un proyecto que involucra a profesores de primaria en el diseño de un serious game a través de la metodología Investigación-Acción junto a la Co-creación. El proyecto da como resultado un juego para promover el trabajo de competencias para el nivel de educación primaria. El juego, llamado "Dolors Aleu", se centra en habilidades básicas para niños de 8-11 años. El artículo presenta el trabajo de investigación realizado por un grupo de investigadores en colaboración con profesores de primaria de Barcelona, España. Argumentamos que la metodología Investigación-Acción y la Co-creación es un primer paso útil para los diseñadores de juegos serios.
\end{abstract}

Palabras-clave: Serious Games, Cocreación, Competencias, Investigación Acción, Educación Primaria

\section{Involving elementary teachers in the design of a serious game through action-research methodology and co-creation}

\begin{abstract}
Serious games are tools that can support school learning. This paper describes a project that involves elementary teachers in the design of a serious game through Action-Research methodology and Co-creation. The project resulted in a serious game to promote work on elementary education level competencies. The game, called "Dolors Aleu", is focused on basic skills for 8-11 year-old children. The paper presents the research work carried out by a group of researchers in collaboration with elementary teachers from Barcelona, Spain. We argue that Action-Research with Co-creation is a useful first step for serious game designers.
\end{abstract}

Keywords: Serious Games, Co-creation, Competences, Action Research, Primary Education 


\section{Introducción}

Desde hace años, las instituciones de educación se han visto inmersas en un proceso de reforma e innovación curricular donde no sólo han tenido que modificar sus planes y programas de estudios, sino que se han visto en la necesidad de transitar hacia un modelo educativo como es el de las competencias (Abreu et al., 2016). Es precisamente en este contexto, donde los serious games pueden ayudar a brindar cambios satisfactorios y donde diversos autores han decidido diseñar juegos porque para los niños, el acto de jugar supone una forma placentera de interactuar con los objetos y sus propias ideas, de tal manera que jugar, implica tratar de comprender el funcionamiento de las cosas (Piaget, Lorenz \& Erikson, 1982). El amplio interés en esta área muestra desde desarrollos teóricos (Gee, 2007; Gibson, 2007) hasta la creación de diversos entornos pensados en una amplia gama de planes de estudios, como el de Riemer \& Schrader (2015) y algunos estudios de caso (Kikot, Fernandes \& Costa, 2015).

Sin embargo, no existe una metodología general para el diseño de juegos educativos. Diferentes autores han abordado propuestas para la creación de juegos como Yusoff, Crowder, Gilbert \& Wills (2009), Kiili (2005), Song y Zhang (2008) o Salen y Zimmerman (2004). Las metodologías o marcos de trabajo propuestos por estos y otros investigadores, tienden a presentar propuestas genéricas. Un ejemplo de ello es el marco de cuatro dimensiones propuesto por De Freitas y Jarvis (2006), citado en varios artículos, y que propone una metodología que no es fácil de utilizar para una amplia gama de contextos educativos. Además de esto los estudios muestran un diseño de investigación que carece de procedimientos; falta información sobre las fases realizadas y la mayoría no se centran en competencias específicas formales que pertenezcan a un currículo. Da la impresión de que faltan medidas que permitan determinar los pasos a seguir.

El grupo de investigación GIE -Grup d'Informàtica a l'Enginyeria- de la Universidad Politécnica de Cataluña diseñó en colaboración con 20 profesores de primaria un juego que incluye en su diseño competencias especificas de aprendizaje. El juego está orientado a alumnos de quinto y sexto grado de primaria y utiliza el currículo de educación de Cataluña. Los profesores influyeron en el proceso de creación porque se han considerado responsables directos de que los juegos lleguen a las aulas. La experiencia dependió por tanto de los usuarios (Kohler, Fueller, Stieger \& Matzler, 2011), y se produjo durante la evaluación de ideas para el juego; la implementación de competencias dentro de la jugabilidad, la narrativa del juego, y el desarrollo del prototipo. Esta actividad de cocreación se realizó bajo la metodología denominada investigación acción. Dada la naturaleza del proyecto, se plantearon los siguientes objetivos:

- Definir la metodología y describir la dinámica de trabajo para el diseño de un serious game;

- Realizar un proceso de cocreación en el cual los profesores de primaria contaran con un papel activo y no pasivo;

- Elegir las competencias que se trabajarían en el juego conjuntamente entre profesores, equipo técnico y de investigación;

- Adaptar el serious game a las necesidades pedagógicas del ciclo superior de primaria, utilizando competencias específicas y manteniendo una jugabilidad atractiva para los alumnos. 
Las principales contribuciones de este artículo son: 1) La metodología investigación acción implementada para diseñar un serious game, 2) La dinámica de trabajo utilizada para generar un proceso de cocreación junto a los profesores, 3) Descripción de las fases y ciclos realizados en el proyecto, 4) El diseño de un serious game directamente relacionado con competencias específicas del currículo de primaria. No se discuten las pruebas de validación hechas sobre el prototipo desarrollado debido a que este texto se centra en explicar una metodología que puede ser replicable para el diseño de un juego educativo.

El presente artículo se encuentra estructurado de la siguiente manera: En la primera sección explicamos las bases teóricas de la propuesta. Se describe la metodología utilizada para desarrollar esta experiencia, así como las diferentes fases y ciclos inmersos en la metodología Investigación-acción en las posteriores. Explicamos la temática del juego, así como las competencias que los alumnos pueden desarrollar utilizando el juego Dolors Aleu. En el antepenúltimo apartado, mencionamos cuales fueron los resultados obtenidos, las limitaciones encontradas en el proyecto y el trabajo futuro. Finalmente, presentamos las conclusiones a las que hemos llegado.

\section{Marco teórico}

\subsection{Educación y competencias}

Cualquier propuesta de acción educativa actual debe enmarcarse en el modelo de competencias debido a que este modelo busca satisfacer las grandes necesidades de la educación actual. Nos referimos a un referente que ha acabado trascendiendo al diseño curricular normativo vigente en España. La Unión Europea estableció una serie de recomendaciones que determinan un marco de referencia de competencias generales, de carácter transversal y que cualquier estudiante debe alcanzar. Estas recomendaciones hacen énfasis en el carácter progresivo del aprendizaje continuo a lo largo de la vida (Kretschmann, 2007), y en contextos abiertos y múltiples. Competencia, por tanto, es un concepto que puede definirse como una combinación de conocimientos, capacidades y actitudes adecuadas al contexto, y que se enmarca en un proyecto de desarrollo personal y colectivo. Es un saber actuar complejo, que se apoya en la combinación eficaz de una variedad de recursos internos y externos dentro de una familia de situaciones (Tardif, 2006).

\subsection{Serious games}

Según la definición de Clark Abt (1970), los serious games tienen un propósito educativo explícito, están cuidadosamente pensados y no están destinados a ser jugados principalmente por diversión. En ellos se deben considerar cuatro componentes estructurales: objetivos, reglas, retos e interacción. El uso de objetivos, reglas y retos en un juego, determinará el orden, los derechos y las responsabilidades de los jugadores. Además, permitirá al jugador enfrentarse a problemas para los cuales tendrá que buscar soluciones. En la interacción, en un juego serio este componente surge de la propia mecánica y dinámica del juego, y da lugar a las vivencias del jugador. Estas experiencias emergen como resultado de la valoración que tendrá el usuario con el juego. Y es esta actividad la que incentivará al alumno a experimentar, a probar múltiples soluciones, a 
descubrir la información y nuevos conocimientos sin temor a equivocarse. Los juegos serios son herramientas innovadoras que se reconocen ampliamente por un potencial considerable para fomentar y apoyar el aprendizaje activo (Romero, Usart \& Ott, 2015). Gracias a un juego de estas características, un estudiante puede desarrollar actitudes positivas hacia los contenidos que muestra, y puede lograr un mayor interés sobre estos al ser tratados en otras actividades fuera del juego (Eguia-Gómez, Contreras-Espinosa \& Solano, 2011) además de que puede contribuir al desarrollo de las habilidades del siglo XXI en la educación. Una disciplina emergente como esta, tiene la responsabilidad de reflexionar críticamente sobre el valor a corto y largo plazo y las consecuencias estructurales de las herramientas que se están desarrollando, promoviendo y utilizando (Mayer, Warmelink \& Zhou, 2015).

\subsection{Proceso de diseño y cocreación}

En un proceso de diseño clásico, los diseñadores generalmente se dedican a realizar actividades analíticas, creativas y de síntesis. De acuerdo con Sanders y Stappers (2014), un proceso de diseño determina el contexto de la innovación y está compuesto por cuatro fases: Pre-diseño, fase de definición de los requisitos de diseño, evaluación del diseño y la fase de introducción del producto en el mercado. Por su parte, un proceso de co-creación inicia con la comprensión de las necesidades de los diferentes actores y objetivos del proyecto. Además, cuando procede, las consecuencias de estas actividades pueden extrapolarse a soluciones de diseño concretas. Según Ali \& Liem (2015), todas las personas son creativas y pueden contribuir al diseño si se les proporcionan un conjunto de herramientas apropiadas. La co-creación ofrece a las redes y a sus actores, oportunidades significativas de innovación, ya que cada actor ofrece acceso a nuevos datos a través de un proceso de integración de recursos (Frow, Nenonen, Payne \& Storbacka, 2015). Nambisan y Nambisan (2008), por su parte, muestran un estudio donde las experiencias de interacción de los usuarios proporcionan un apoyo empírico para afirmar que son los usuarios quienes aportan valor a la cocreación. Para ello proponen un marco analítico y sugieren cuatro dimensiones para los sistemas de cocreación: la pragmática, la sociabilidad, la hedónica y la usabilidad. El objetivo no es otro que atender a las necesidades de los participantes. La dimensión denominada "pragmática" se refiere a la experiencia del usuario en la realización de objetivos relacionados con el producto en sí, aquel que le permite estar informado e inspira su creatividad. Los aspectos sociales, por su parte, se refieren a las relacionales subyacentes de estas interacciones y conforman el componente "sociabilidad". El siguiente punto, se relaciona con la interacción dada en espacios virtuales, con lo que se hace referencia al componente "hedónico". Finalmente, la dimensión "usabilidad", se destina a observar la calidad de las interacciones del ser humano con la máquina. Estos componentes sirven como un marco básico para iniciar un proceso de cocreación, pero se han adaptado a nuestras necesidades particulares. Es decir, anteriormente estas estrategias no se han utilizado en espacios interactivos como juegos digitales y por ello hemos decidido renombrar la dimensión “Usabilidad”. Un juego envuelve muchas más métricas que un espacio interactivo como un foro o web. Los valores que determinan las experiencias de los usuarios tienen un alto impacto en la capacidad para transformar ideas y requerimientos de los usuarios en productos y servicios. La dimensión se ha denominado "Experiencia del Usuario”. La Tabla 1 enumera los componentes. 


\begin{tabular}{ll} 
Componente de experiencia & \multicolumn{1}{c}{ Características } \\
\hline Pragmatismo & $\begin{array}{l}\text { Los participantes reciben información dirigida a impulsar y } \\
\text { preparar el escenario para las siguientes actividades. Se enfrentan } \\
\text { con material para estimular la creatividad. }\end{array}$ \\
\hline Sociabilidad & $\begin{array}{l}\text { Se fomenta la colaboración limando las expectativas de los } \\
\text { participantes. Para nutrir debates y colaboración, la discusión } \\
\text { comienza cuando los usuarios se sienten preparados. }\end{array}$ \\
\hline Hedonismo & $\begin{array}{l}\text { Participar en un proceso de cocreación es un desafío estimulante } \\
\text { para los participantes. }\end{array}$ \\
\hline Experiencia de usuario & $\begin{array}{l}\text { Los participantes experimentan el proceso. Dan opiniones en } \\
\text { base a una propuesta prediseñada por el grupo de investigación. }\end{array}$ \\
\hline
\end{tabular}

Tabla 1 - Componentes de experiencia. Fuente: Adaptada de Eguia-Gómez, Contreras-Espinosa y Solano (2014).

\section{Metodología utilizada}

Esta investigación se enmarca en la investigación-acción, en adelante IA. Para el diseño del juego, partimos de una experiencia previa (Contreras-Espinosa \& Eguia-Gómez, 2016), además de una amplia revisión bibliográfica y del análisis de otros juegos existentes en el mercado. La IA tiene como objetivo capitalizar el aprendizaje conjunto entre el investigador y los sujetos inmersos en el contexto (Susman, \& Evered, 1978) y es especialmente adecuada cuando los investigadores tienen que involucrarse para dotar de valor a un proyecto, pero dentro del problema, sobre todo cuando el proceso de cambio en sí es el tema que se está estudiando (Davison, 1999). En la literatura la IA ha sido descrita como el proceso más adecuado para desarrollar los principios de diseño de un sistema (Lindgren, Henfridsson, \& Schultze, 2004) y, dado que el propósito de este estudio es crear los principios de un serious game, se ha tenido en cuenta la participación de profesores de primaria como actores principales.

El proyecto se desarrolló a lolargo de 12 meses que empezaron en el mes de septiembre de 2013. Se seleccionaron 20 profesores de escuelas primaria del área metropolitana de Barcelona. La elección de los participantes dependió de su disposición a trabajar en el proyecto, su creatividad y participación en experiencias previas similares. Mediante grupos de discusión, se pudieron obtener sus recomendaciones y experiencias utilizando juegos en las aulas. El tiempo asignado para cada grupo dirigido fue de dos horas aproximadamente. El grupo de profesores contaba con una edad de entre 25 y 50 años. Se invitó a los participantes a entrar en el contexto del problema, a inspirarse y participar en los retos que el juego podía plantear a sus estudiantes, además de aportar conceptos e intercambiar ideas con los investigadores.

\subsection{Primer ciclo de la IA}

Durante el primer ciclo se realizaron cinco fases. Como en todo proceso, la IA pasa por fases (Davison, 1999). En nuestro caso, se realizaron dos ciclos divididos en las siguientes fases: el diagnóstico, la planificación de la acción, actuación, evaluación y especificaciones de aprendizaje. 
Fase I. El diagnóstico. Con la intención de identificar experiencias en los niveles tecnológico, pedagógico y creativo, se partió de una revisión bibliográfica y de un análisis de juegos existentes en el mercado. Se formuló la hipótesis: Es posible realizar un proceso de cocreación en el diseño de un serious game que tiene como objetivo desarrollar competencias de aprendizaje.

Fase II. Planificación de la acción. Se planificó el proceso de cocreación y más tarde se afinó una propuesta teniendo en cuenta la literatura científica. Los principios se adaptaron a necesidades particulares:

a) Pragmatismo: Desarrollar elementos interactivos para situar a los participantes e inspirar su creatividad; b) Sociabilidad: Fomentar la colaboración; c) Hedonismo: Proporcionar tareas desafiantes; d) Experiencia de usuario: Simplificar la experiencia de los participantes y proporcionar una experiencia grata.

Más tarde, se planificaron los grupos de discusión.

Fase III. Acción. Para iniciar con el proceso, una primera idea se discutió con todos los profesores. En grupos de discusión se habló de recomendaciones y experiencias haciendo hincapié en el poder disuasorio de los juegos como mundos virtuales. Se concretó la temática del juego y el personaje principal que serviría de avatar. Se pidió a los profesores que expresaran sus ideas y experiencias para participar en el diseño del juego. Los participantes fueron capaces de valorar, comentar y juzgar ideas.

Fase IV. Evaluación. La evaluación se centró en los resultados obtenidos, que permitieron comprender los requisitos necesarios para que el juego fuera útil e instructivo. Los profesores son quienes mejor pueden describir las concepciones subjetivas.

Fase V. Especificaciones. El equipo de investigación registró las fases. En base a ello, realizó un informe.

Gracias a esta experiencia previa, pudimos refinar el proceso llevado a cabo en una investigación anterior en el año 2012 (Contreras-Espinosa \& Eguia-Gómez, 2016). El marco teórico nos orientó en el diseño y nos proporcionó una valiosa comprensión inicial sobre las cuestiones generales. Nos embarcamos por tanto en el segundo ciclo de IA recurriendo al informe del primer ciclo, pero con una nueva fase de diagnóstico.

\subsection{Segundo ciclo de la IA}

A continuación, se describen las cinco fases del segundo ciclo:

Fase I. El diagnóstico. Con la intención de preparar el diseño del juego, se identificaron los principios de diseño y se observaron tendencias a nivel tecnológico, pedagógico y creativo. Se unieron los resultados de la revisión bibliográfica, el análisis de juegos existentes en el mercado y los resultados de los grupos de discusión. Se tomaron en consideración los cuatro componentes estructurales en un serious game mencionados por Abt (1970).

Fase II. Planificación de la acción. Se definieron los requisitos necesarios para la creación de un juego útil e instructivo. El equipo inició de esta forma un documento de diseño que indicaba las reglas del juego, las mecánicas, los personajes, la jugabilidad y las competencias inmersas en el mismo. Se decidió, además, la programación del juego con el fin de garantizar la interoperabilidad para la comunidad a la cual va dirigido. 
Fase III. Acción. Se elaboró una primera propuesta acorde con las competencias y procesos de cocreación llevados a cabo en el ciclo anterior. Se diseñó un prototipo para realizar pruebas en una posterior fase.

Fase IV. Evaluación. Se realizaron pruebas de usabilidad y se recopilaron los resultados obtenidos.

Fase V. Especificaciones. Se registraron las especificaciones, las fases y los ciclos.

Los resultados de validación no se describen en este artículo por cuestiones de espacio.

En la Tabla 2 se resumen las ocho fases realizadas en los dos ciclos del proyecto:

\begin{tabular}{|c|c|}
\hline Ciclo 1 & Ciclo 2 \\
\hline \multicolumn{2}{|l|}{ Fase I. Diagnóstico } \\
\hline $\begin{array}{l}\text {-Identificación de las posibilidades y alcances de } \\
\text { la IA. } \\
\text {-Revisión de literatura asociada. La búsqueda } \\
\text { se restringió a artículos relacionados con las } \\
\text { palabras clave: serious games, competences, } \\
\text { action research y co-creation. } \\
\text {-Análisis de productos existentes en el mercado, a } \\
\text { nivel tecnológico, pedagógico y creativo. }\end{array}$ & $\begin{array}{l}\text {-Desarrollo de una propuesta tomando en cuenta los } \\
\text { resultados de los grupos de discusión, los resultados } \\
\text { de la revisión bibliográfica, y el análisis de los } \\
\text { productos existentes en el mercado. Utilización de } \\
\text { técnicas de brainstorming, collages y testeos } \\
\text { de juegos. }\end{array}$ \\
\hline
\end{tabular}

\section{Fase II. Planificación de la acción}

-Planificación del proceso de cocreación.

-Planificación de los grupos de discusión virtuales

-Definición de los requisitos necesarios para un con profesores que utilizan juegos digitales.

juego serio útil e instructivo enfocado a estudiantes catalanes.

-Decisión sobre la tecnología a utilizar con el fin de garantizar la interoperabilidad de los juegos para la comunidad a la cual va dirigido el proyecto.

-Analisis de los dispositivos utilizados habitualmente por los alumnos de primaria en las aulas catalanas.

-Desarrollo del documento de diseño para el juego.

\section{Fase III. Acción}

-Desarrollo de 3 grupos de discusión

semiestructurados con una duración de 2 horas.

-Población de 25 docentes con edades entre los 24 y los 52 años.
-Diseño de un prototipo, estableciendo reglas del juego, mecánicas, personajes, jugabilidad del juego, y competencias de aprendizaje.

\section{Fase IV. Evaluación}

-Resultados de los grupos.

-Pruebas de usabilidad.

-Análisis de los resultados obtenidos en los grupos -Resultados y análisis de las pruebas. de discusión.

\section{Fase $V$. Especificaciones}

-Registro de las fases y ciclo 1.

-Registro de las fases y ciclo 2. Planeación de la introducción del juego en las aulas.

Tabla 2 - Fases y ciclos del proyecto. Fuente: Adaptada de Eguia Gómez, Contreras-Espinosa y Solano (2014).

Posteriormente se estudiaría la introducción del juego en las aulas. Estos resultados no se describen aquí por no ser el objetivo de este artículo. 


\section{Descripción del serious game}

Como resultado del proceso de cocreación presentamos el juego destinado a niños de 8 a 11 años desarrollado con Unity. El objetivo de este juego es dar a conocer un personaje histórico y su relación con los avances en el campo de la medicina durante la segunda mitad del siglo XIX. Dolors Aleu i Riera fue la primera mujer licenciada en medicina en Cataluña y la segunda que alcanzó el título de doctor. Tras doctorarse, un proceso que se alargó mucho debido a impedimentos por el hecho de ser mujer, se especializó en ginecología y medicina infantil. Trabajó como médico durante 25 años, y realizó tareas de divulgación de buenos hábitos higiénicos, especialmente entre la población femenina, en la Academia para la Ilustración de la Mujer, institución que fundó. Las acciones que el jugador debe realizar son intuitivas, y una narración le proporciona instrucciones sobre lo que tiene que hacer y cómo hacerlo. El jugador recibe ayuda constante mediante texto e imágenes que muestran lo que está sucediendo. El juego sigue la estructura de los juegos de plataformas, una tipología de éxito entre jóvenes, según lo observado en el análisis de los juegos existentes en el mercado. La estructura del juego incluye 5 niveles con actividades que conforman la narración lineal. En la Tabla 3, puede verse el orden de cada nivel. El juego está diseñado para ser utilizado en una plataforma web, tomando en cuenta que los dispositivos más utilizados en las aulas de Barcelona son ordenadores.

\begin{tabular}{ll}
\hline Nivel & \multicolumn{1}{c}{ Nombre } \\
\hline 1 & La Universidad. Año 1874. \\
\hline 2 & Ciudad Vella. Año 1875. \\
\hline 3 & Hospital de la Santa Cruz. Año 1876. \\
\hline 5 & Plaza Cataluña. Año 1882. \\
\hline
\end{tabular}

Tabla 3 - Niveles del Prototipo Dolors Aleu. Fuente: Propia.

\subsection{Niveles definidos en el juego}

Cada nivel trata un tema concreto relacionado con la vida y el entorno de Dolors Aleu. El jugador accederá al juego no sin antes observar una introducción que le servirá para situarlo temporalmente en la historia. Finalizada la introducción, podrá empezar a superar los primeros niveles del juego, que son de dificultad creciente y explican la dinámica del mismo. Se busca simplificar la experiencia del usuario proporcionando una navegación intuitiva. Figura 1.

En el primer nivel titulado "La universidad" Dolors muestra su deseo de ir a la universidad. En este nivel encontrará diferentes personajes que intentarán desanimarle a estudiar medicina y le ponen a prueba. El nivel permite reflexionar sobre los derechos de las personas y la discriminación invitando al jugador a superar el reto. En el nivel "Ciudad Vella", habrá pasado un año desde que Dolors entró en la universidad y ha podido mostrar su valía. Se encuentra con otra estudiante que le pide ayudar a gente que necesita atención médica. A través de los personajes, conocerá la historia de la ciudad y su arquitectura. Más adelante, cuando ha de ayudar a personas que padecen diferentes problemas de salud, se enfrentará con la necesidad de saber como combatir enfermedades 


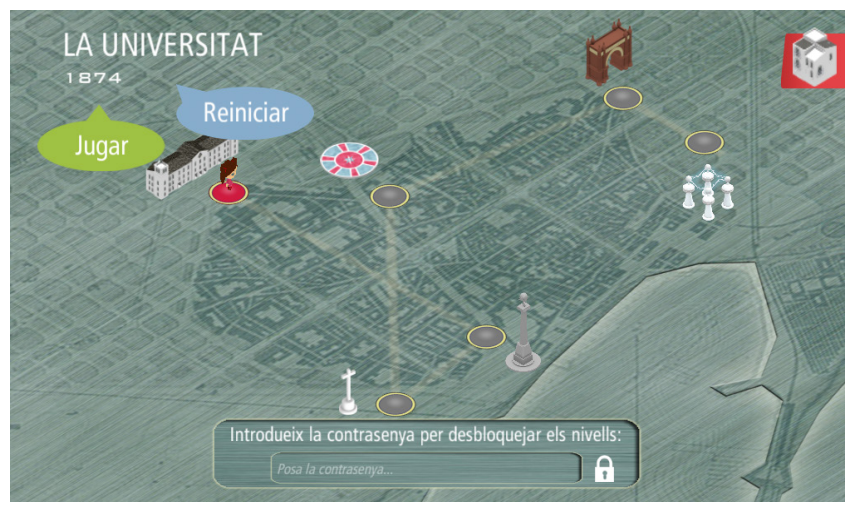

Figura 1 - Interfaz gráfica. Fuente: Dolors Aleu. Colección Personatges en Joc.

de aquella época, muchas de ellas, consecuencia de tener una dieta insuficiente y poco equilibrada, así como la falta de hábitos higiénicos. Para este caso se ha planeado un juego paralelo de cartas que muestran como combatir enfermedades. Figura 2.

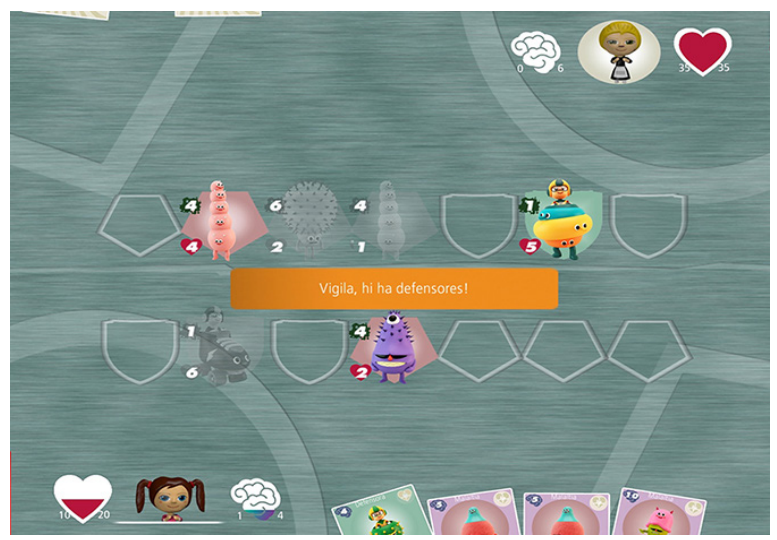

Figura 2 - Juego de cartas para combatir enfermedades. Fuente: Dolors Aleu. Colección Personatges en Joc.

En el nivel, "Hospital de la Santa Cruz", la protagonista deberá recorrer diferentes habitaciones donde recibirá explicaciones sobre los diferentes pasos del método científico. En cada una de ellas le propondrán hacer una prueba para demostrar que ha entendido el método. En el nivel 4, Dolores Aleu terminará los estudios en 1879, pero no le permitirán hacer el examen de licenciatura. Le pedirán que vaya al Ateneo Obrero a dar una conferencia sobre los problemas de higiene pública. Una vez más, debe convencer a la gente de que es médico, y hablará con estudiantes sobre los movimientos obreros y las manifestaciones más destacadas de la época. En el nivel cinco, Dolors se sitúará en el mismo año en que se llevó a cabo la Exposición Universal. Es alertada de un problema de salud pública, donde mucha gente sufrirá de una intoxicación alimentaria. Se sospecha que una persona está intentando boicotear la exposición. En la exposición 
conocerá nuevos inventos, como la energía eléctrica. Más adelante descubrirá que el Dr. Moreau es el causante de muchos de los problemas de la ciudad. Debe enfrentarse a él con inventiva. Este nivel da pie para que los alumnos piensen algún invento, así como los pasos que seguirían para conseguir la fabricación.

\subsection{Competencias presentes en el juego}

Se aprovecha la jugabilidad para trabajar diversos aspectos del currículo que pueden ampliarse en el aula. Los contenidos forman parte de las reglas del juego, y la dinámica del mismo consiste en mostrar información estrechamente relacionada con las competencias del currículo. Las competencias básicas trabajadas en el juego se describen a continuación. Dimensión comprensión lectora y audiovisual.

1. Leer con fluidez para comprender textos de la vida cotidiana, de los medios de comunicación y escolares en diferentes formatos y soportes.

2. Aplicar estrategias de comprensión para obtener información, interpretar y valorar el contenido de acuerdo con la tipología y la complejidad del texto.

3. Utilizar, para comprender un texto, estructura y formato de cada género textual y de las estructuras morfosintácticas más habituales.

4. Aplicar estrategias de búsqueda y gestión de la información para adquirir conocimiento propio.

El juego desarrolla la competencia lingüística con la lectura de diversa información necesaria para ir avanzando. De esta forma se aprenden aspectos históricos y geográficos.

Las competencias básicas del ámbito matemático y que son trabajadas con los alumnos se describen. Dimensión resolución de problemas:

1. Traducir un problema a una representación matemática y emplear conceptos, herramientas y estrategias matemáticas para resolverlo.

2. Dary comprobar la solución de un problema de acuerdo con las preguntas planteadas;

3. Hacer preguntas y generar problemas de carácter matemático.

Dimensión razonamiento y prueba:

4. Hacer conjeturas matemáticas adecuadas en situaciones cotidianas y comprobarlas.

5. Argumentar las afirmaciones y los procesos matemáticos realizados en contextos próximos.

Dimensión conexiones:

6. Establecer relaciones entre diferentes conceptos, así como entre los diversos significados de un mismo concepto.

7. Identificar las matemáticas implicadas en situaciones cotidianas y escolares y buscar situaciones que se puedan relacionar con ideas matemáticas concretas.

8. Expresar ideas y procesos matemáticos de manera comprensible utilizando el lenguaje verbal (oral y escrito).

9. Usar las diversas representaciones de los conceptos y las relaciones para expresar matemáticamente una situación. 
10. Usar las herramientas tecnológicas con criterio, de forma ajustada a la situación, e interpretar las representaciones que ofrecen.

En el juego se plantean algunos problemas matemáticos relacionados con situaciones cotidianas. El juego también prevé que los usuarios tengan que hacer cálculos para intentar ganar al adversario.

Finalmente, las competencias básicas del ámbito digital. Dimensión Instrumentos y aplicaciones:

1. Seleccionar, utilizar y programar dispositivos digitales y sus funcionalidades de acuerdo con las tareas que deben llevarse a cabo.

Dimensión: tratamiento de la información y organización de los entornos de trabajo y de aprendizaje

2. Buscar, contrastar y seleccionar información digital considerando diversas fuentes y entornos digitales.

3. Construir nuevo conocimiento personal mediante estrategias de tratamiento de la información con el apoyo de aplicaciones digitales.

Se potencia el aprendizaje de habilidades y estrategias en tecnologías digitales. Los usuarios, además del juego, también pueden visitar otros sitios web donde podrán aprender más cosas relacionadas con el contexto y el momento histórico en que se desarrolla el videojuego. Las competencias consideran importante tener ejes procesuales para facilitar el establecimiento de criterios por un determinado desempeño en concreto. En los criterios aparecen desglosados los diversos saberes de una competencia. Los contenidos ya no son estáticos, son saberes dinámicos. De esta forma los contenidos cobran vida en la resolución de actividades y problemas.

\section{Discusión}

Sintetizar los conocimientos adquiridos a partir de un estudio de IA que involucra a diferentes estrategias e intervenciones de recolección de datos, genera un conjunto de principios de diseño que pueden aplicarse a un sistema de cocreación. El seguir una metodología tan extendida en diferentes áreas, nos ha permitido pautar y describir una dinámica de trabajo que puede ser útil para el diseño de un serious game dentro de un amplio contexto educativo. En general, los modelos, métodos, metodologías, tareas y estrategias existentes, así como otras formas de apoyo, no conforman una base clara. Autores como Van Rosmalen, Wilson \& Hummel (2013) señalan, que existe poco material para obtener ayuda, y a pesar de que diversas propuestas actuales proporcionan interesantes conceptos, falta definir su aplicación práctica. No existe una metodología general para el diseño de juegos educativos; las metodologías y marcos de trabajo propuestos son propuestas genéricas o poco replicables y que muestran un diseño de investigación sin procedimientos. Contamos con marcos que proporcionan algunas directrices, pero se consideran un trabajo aun en proceso (Dondlinger, 2007). Sin embargo, las pautas aquí indicadas permiten realizar el diseño de una propuesta utilizando dos ciclos divididos en las fases de diagnóstico, planificación de la acción, actuación, evaluación y especificaciones de aprendizaje. Del análisis de los juegos 
existentes en el mercado, podemos concluir que son juegos que no están enfocados en desarrollar unas competencias formales específicas. Por ejemplo, es el caso de juegos como Lure of the Labyrinth, Cool School o WolfQuest. En cuanto a algunos materiales interactivos detectados en la red Edu365.cat, creados por profesores, instituciones y otros autores del ámbito de la educación, estos están creados con la intención de alinearse a áreas específicas de un currículo formal, pero no cuentan con los cuatro componentes estructurales de un serious game descritos por Abt (1970). La evaluación permitió comprender los requisitos necesarios para diseñar el juego y que este fuera útil e instructivo. En cuanto a las experiencias detectadas en publicaciones científicas, si bien se centran en mostrar competencias específicas formales que forman parte de algunos juegos digitales educativos, pero no se describe el proceso de diseño que fue utilizado. Una vez registradas las fases y tomado en cuenta los puntos desarrollados el informe nos sirvió para analizar el conocimiento y la puesta en marcha del proceso de cocreación y en que fases. El marco teórico nos orientó en el proceso, pero concluimos que es necesario buscar una visión más profunda con el fin de ayudar a los diseñadores. Como resultado, la Tabla 4, ilustra las diferentes fases de diseño descritas por Sanders y Stappers (2014), en que momento pueden ser abordadas en las fases y ciclo del proceso de cocreación, y en que estado debe encontrarse la propuesta del juego serio. En la última fase se estudiaría la introducción del juego en las aulas, y en este caso se utilizaría la versión final del juego.

\begin{tabular}{lcc}
\hline Fases de diseño & Fase del ciclo & Estado del juego \\
\hline Pre-diseño & Fase 2, ciclo 2 & Documento de diseño \\
\hline Requisitos de diseño & Fase 3, ciclo 2 & Prototipo \\
\hline Evaluación del diseño & Fase 4, ciclo 2 & Prototipo \\
\hline Introducción del producto & Nuevo ciclo & Juego Beta \\
\hline
\end{tabular}

Tabla 4 - Fases de diseño, cocreacion y estado del juego. Fuente: Propia.

Hemos comprobado, además, que estos principios pueden contribuir a facilitar el diseño de un serious game, pero, además, contribuyen a la literatura que se centra en el diseño de juegos educativos, cuando se recomienda utilizar cocreación con el fin de innovar y con la idea de incluir a los usuarios (Von Hippel, 2005; Kangas, 2010; Nambisan \& Nambisan, 2008; Mouws \& Bleumers, 2015).

Al utilizar la cocreación se ponen de manifiesto incluso las necesidades reales de los usuarios, en este caso los profesores, que son quienes utilizaran los juegos en las aulas posteriormente. Si la experiencia satisface a sus necesidades hedónicas, los esfuerzos realizados habrán valido la pena, porque los usuarios finales serán colectivamente capaces de resolver problemas que antes no podrían haber superado, tal como deduce Von Ahn (2008). Con ello, los profesores se apropian del producto generado lo que facilita el uso de los juegos en las aulas. Debemos implicar a los profesores en el diseño de juegos educativos, ya que esta demostrado que los estudiantes que han recibido formación mediante juegos serios, están más dispuestos a colaborar y establecer acciones de trabajo en equipo, gracias a la motivación adicional que 
supone la resolución de un desafío (Poy-Castro, Mendaña-Cuervo \& González, 2015). En cuanto a utilizar los componentes definidos por Nambisan y Nambisan (2008), nuestra recomendación es utilizarlos como una base, sobre todo el componente de Pragmatismo, Sociabilidad y Hedonismo. Sin embargo, el componente de Usabilidad debe modificarse para poder trabajar con serious games. En el modelo de Nambisan y Nambisan este es un punto crucial para la creación de la arquitectura e infraestructura de un producto en línea, pero nuestra aportación al modelo, es incluir en este punto la experiencia del usuario, lo que incluye directamente el diseño de mecánicas y dinámicas de juego, así como discutir una primera aproximación sobre cuales serán los desafíos que deberán de ajustarse a las habilidades de los jugadores. Consideramos que otros investigadores pueden replicar esta propuesta siguiendo los pasos descritos con la metodología IA. El desarrollo de esta metodología ha permitido, además: a) Definir el perfil de los usuarios y sus características; b) Elegir las competencias que se trabajarán en un posterior juego; c) Garantizar la interoperabilidad del juego teniendo en cuenta los dispositivos utilizados habitualmente por los alumnos en las aulas catalanas tomando en cuenta el tipo de ordenadores y, en algunos casos, el uso de dispositivos móviles. En cuanto a las necesidades futuras de investigación, y como ya lo expresamos anteriormente, otras sesiones de cocreación ayudarán a refinar el método utilizado. Es de vital importancia diseñar un sistema con un enfoque que permita diseñar y evaluar juegos con la ayuda de los alumnos de primaria, sin embargo, consideramos que es primordial empezar con una propuesta enfocada a las personas que hacen llegar los juegos a las aulas: los profesores.

\section{Conclusión}

En esta investigación se propuso utilizar la metodología Investigación-Acción junto a un proceso de cocreación para crear un serious game en donde los profesores de primaria tuvieron un papel activo en el diseño del juego. La IA es útil y puede replicarse fácilmente en proyectos cuyo fin sea diseñar un serious game. La dinámica de trabajo utilizada para generar un proceso de cocreación junto a los profesores definió todo el proceso. Por tanto, fue posible demostrar que para configurar adecuadamente un proceso de cocreación es necesario contar con metodologías estandarizadas. Además, y como uno de los objetivos, se han descrito las diferentes fases y ciclos del proyecto, que nos permitieron elegir las competencias que se trabajarían posteriormente en un serious game en base a las necesidades pedagógicas específicas del ciclo superior de primaria. Resaltamos que el trabajo en un equipo multidisciplinar es enriquecedor y necesario, pero plantea la necesidad de conjugar diferentes puntos de vista, objetivos y prioridades en su desarrollo. La comunicación constante en proyectos de estas características es fundamental para realizar un trabajo multidisciplinar donde cada profesional debe aportar su conocimiento y experiencia en la consecución de los objetivos.

\section{Agradecimientos}

Agradecemos al Comissionat per a Universitats i Recerca de la Generalitat de Catalunya la financiación otorgada para la realización de este juego. 


\section{Referencias bibliográficas}

Abreu, A., Rocha, Á., de Carvalho, J. V., \& Cota, M. P. (2016). The electronic booklet on teaching-learning process: Teacher vision and parents of students in primary and secondary education. Telematics and Informatics, August 12. http://dx.doi.org/10.1016/j.tele.2016.08.011

Abt, C. (1970). Serious Games. New York: Viking Press

Ali \& Liem (2015). The use and value of different co-creation and tools in the design process. En: Proceedings of the 2oth International Conference on Engineering Design (ICED15), 1-10, 27-30 July 2015.

Contreras-Espinosa, R. \& Eguia-Gómez, J. (2016). Investigación-acción como metodología para el diseño de un serious game. RIED, 19(2), 71-90. doi: http://dx.doi.org/10.5944/ried.19.2.15624

Davison, R. (1999). GSS and Action Research in the Hong Kong Police. Information Technology \& People, 14(1), 60-77. doi: http://dx.doi. org/10.1108/09593840110694984

De Freitas, S. \& Jarvis, S. (2006). A Framework for Developing Serious Games to meet Learner Needs. Interservice/Industry Training, Simulation and Education Conference, Orlando, Florida.

Dondlinger. M.J. (2007). Educational video game design: A review of the literature. Journal of Applied Educational Technology, 4 (1), 21-31.

Eguia-Gómez, J., Contreras-Espinosa, R. \& Solano, L. (2011). Videojuegos como un entorno de aprendizaje: el Caso de Monturiol el joc. Icono 14, 9(2), 249-261. doi: http://dx.doi.org/10.7195/ri14.v9i2.35

Eguia-Gómez, J., Contreras-Espinosa, R. \& Solano, L. (2014). Co-creation of a serious game to develop competences on the primary education program, Information Systems and Technologies (CISTI 2014) 9th Iberian Conference, 1-6, 18-21 June 2014. doi: http://ieeexplore.ieee.org/ document $/ 6877080 /$ ?arnumber $=6877080$

Frow, P., Nenonen, S., Payne, A. \& Storbacka, K. (2015). Managing Co-creation Design: A Strategic Approach to innovation. British Journal of Management, 26, 463-483. doi: $10.1111 / 1467-8551.12087$

Gee, J.P. (2007). What video games have to teach us about learning and literacy. New York: Palgrave Macmillan.

Gibson, D. , Aldrich, C. \& Prensky, M. (2007). Games and simulations in online learning: Research and development frameworks. Hershey: Information Science Publishing.

Kangas, M. (2010). Creative and playfull learning: Learning through game co-creation and games in a playful learning environment. Thinking Skills and Creayivity, 5(1), 1-15. doi: http://dx.doi.org/10.1016/j.tsc.2009.11.001 
Kiili, K. (2005). Content creation challenges and flow experience in educational games: The IT-Emperor case. The Internet and Higher Education, 8(3), 183-198. doi:10.1016/j.iheduc.2005.06.001

Kikot, T., Fernandes, S. \& Costa, G. (2015). Potencial da aprendizagem baseada-emjogos: Um caso de estudo na Universidade do Algarve. RISTI - Revista Ibérica de Sistemas e Tecnologias de Informação, 16(12), 17-29. doi: http://dx.doi. org/10.17013/risti.16.17-29

Kohler, T., Fueller, J., Stieger, D. \& Matzler, K. (2011). Avatar-based innovation: consequences of the virtual co-creation experience. Computers in Human Behavior, 27 (1), 160-168. doi: http://dx.doi.org/10.1016/j.chb.2010.07.019

Kretschmann, R. (2007). Developing competencies by playong digital sports-games. USChina Education Review, 7(2), 67-75. doi: http://dx.doi.org/10.5430/wje.v1n1p3

Lindgren, R., Henfridsson, O. \& Schultze, U. (2004). Design Principles for Competence Management Systems: A Synthesis of an Action Research Study. MIS Quarterly, 28 (3), 435-472.

Marton, F. (1981). Phenomenography: Describing Conceptions of the World Around Us. Instructional Science, 10(2), 177-200. doi: http://dx.doi.org/10.1007/BFo0132516

Mayer, I., Warmelink, H. \& Zhou, Q. (2015). A frame-reflective discourse analysis of serious games. British Journal of Educational Technology, 47(2), 342-357. doi: 10.1111/bjet.12245

Mouws, K. \& Bleumers, L. (2015). Co-creating Games with Children: A case Study. International Journal of Gaming and Computer-Mediated Simulations, 7(3), 22-43. doi: http://dx.doi.org/10.4018/IJGCMS.2015070102

Nambisan, P. \& Nambisan, S. (2008). How to Profit from a better 'Virtual Customer Environment'. MIT Sloan Management Review, 49(3), 53-61. doi: http://dx.doi. org/10.1225/SMR279

Piaget, J., Lorenz, K. \& Erikson, E. (1982). Juego y desarrollo. Madrid: Grijalbo.

Poy-Castro, R., Mendaña-Cuervo, C. \& González, B. (2015). Diseño y evaluación de un juego serio para la formación de estudiantes universitarios en habilidades de trabajo en equipo. Revista Ibérica de Sistemas e Tecnologias de Informação, 3(3), 71-83. doi: http://dx.doi.org/10.17013/risti.e3.71-83

Riemer, V. \& Schrader, C. (2015). Learning with wuizzes, simulations, and adventures: Student's attitudes, perceptions and intentions to learn with different types of serious games. Computers \& Education, 88, 160-168. doi: http://dx.doi. org/10.1016/j.compedu.2015.05.003

Romero, M., Usart, M. \& Ott, M. (2015). Can Serious Games Contribute to developing and dustaining 21st Century Skills?. Cultural Studies, 10(2), 148-177. doi: 10.1177/1555412014548919

Salen, K. \& Zimmerman, E. (2004). Rules of Play: Game Design Fundamentals. Cambridge, Massachusetts: The MIT Press. 
Sanders. E. \& Stappers P. J. (2014). Probes, toolkits, and prototypes: Three approaches to making in codesigning. CoDesign, 10(1), 5-14. doi: 10.1080/15710882.2014.888183

Song, M. \& Zhang, S. (2008). EFM: A Model for Educational Game Design, Technologies for E-Learning and Digital Entertainment, 5093, 509-517, 2008. doi:10.1007/9783-540-69736-7_54

Susman, G. I. \& Evered, R. D. (1978). An Assessment of the Scientific Merits of Action Research. Administrative Science Quarterly, 23(4), 582-603.

Tardif, J. (2006). L'evaluation des compénces. Documenter le parcours de développement. Montréal: Chenelière Éducation.

Van Rosmalen, P., Wilson, A. \& Hummel, H. (2013). Games for and by teachers and learners. En Connolly, T.M., Boyle, E., Hainey, T., Baxter, G. \& Moreno-Ger, P. (Eds), Psychology, Pedagogy and Assessment in Serious Games (pp. 243-269), IGI Global.

Von Ahn, L., Maurer, B., McMillen, C., Abraham, D. \& Blum, M. (2008). reCAPTCHA: Human-Based Character Recognition via Web Security Measures. Science, 321, 1465-1468. doi: http://dx.doi.org/10.1126/science.1160379

Von Hippel, E. (2005). Democratizing Innovation. Cambridge: The MIT Press.

Yusoff, A., Crowder, R., Gilbert, L. \& Wills, G. (2009). A Conceptual Framework for Serious Games, The 9th IEEE International Conference on Advanced Learning Technologies (ICALT 2009), 21-23, 15-17 July 2009. doi: 10.1109/ICALT.2009.19 